to bacterial vaginosis (BV), a risk for HIV acquisition. Limited research has focused on IVPs and their correlates among adolescent girls and young women (AGYW) in South Africa, a key population for HIV prevention efforts.

Methods We used cross-sectional baseline survey data from 253 HIV-negative or HIV-status unknown AGYW (16-24 years) enrolled in AYAZAZI, a community-based cohort study in Soweto and Durban, South Africa (2014-2016). We measured IVP use in the past 30 days for 11 IVPs (yes vs no) and estimated the prevalence of using $\geq=1$ IVP in the past 30 days (yes vs no). Using existing literature, we identified 18 determinants of IVP use. We estimated the total causal effect of each determinant on any IVP use in the past 30 days using separate logistic regression models adjusted for confounding within the counterfactual framework of causal inference.

Results Mean age was 19 years, 67.2\% were in school, and $81.8 \%$ ever had sex. Overall IVP prevalence was $76.7 \%(59 \%$ vs $81 \%$ among those who had never vs ever had sex; $\mathrm{p}=0.016)$. The most common IVPs included: washing intravaginally with water only (66\%), with soap (41\%), and with other products including towels or sponges (41\%). AGYW who reported binge drinking $(\mathrm{aOR}=7.6$; 95\%CI: 3.2-18.4), a higher monthly income $(\geq \mathrm{R} 1601$ vs $\leq \mathrm{R} 400$; aOR $=7.4$; $95 \%$ CI: 1.6-33.9), engaged in transactional sex $(\mathrm{aOR}=4.4 ; 95 \%$ CI: 1.0-19.1), and/or reported any symptoms of genital tract infections $(\mathrm{aOR}=4.9$; 95\%CI: 1.0-23.0) had higher adjusted odds of using IVPs.

Conclusion Over three-quarters of South African AGYW in this study reported IVPs with differences by socio-economic, behavioral, and clinical characteristics. Further investigation of IVP motives and implications for BV and HIV acquisition risk among young women are warranted.

Disclosure No significant relationships.

\section{P398 CAN VIDEO-CONSULTATIONS CONTRIBUTE TO DIMINISHING WAITING LISTS? A DEMONSTRATION PROJECT IN THE NETHERLANDS}

${ }^{1}$ Filippo Zimbile*, ${ }^{2}$ Rik Crutzen. ' Soa AIDS Nederland (STI AIDS The Netherlands), Youth, Amsterdam, Netherlands; ${ }^{2}$ Maastricht University, Health Promotion, Maastricht, Netherlands

\subsection{6/sextrans-2019-sti.490}

Background The 25 Centers for Sexual Health (CSHs) in The Netherlands offer free STI and Sexual Health consultations to adolescents: annually approximately 70.000 STI and 11.000 Sexual Health consultations. In some regions there are waiting times for the consultations. Furthermore, the reach of regular consultations is limited among lower educated young people. In a joint pilot project the CSHs wanted to gain insight into whether online video-consultations can contribute to solving these problems. In the first phase of this project, the key question is whether a video consultation is appreciated and can offer sufficient quality, and - if so - for which services and for which target groups? We also explored the time periods of the video consultations.

Methods The key question is approached from two perspectives: client and professional. In 8 regions CSHs offered video consultations - including STI testing methods on distance - to adolescents for whom no physical examination was necessary. Both clients and professionals filled out an online questionnaire directly after the (video)consultation. 20 adolescents were interviewed, all professionals participated in regional organized focusgroups. To create a reference frame, regular consultations were evaluated in the same way. The software of the video-consultations registered time periods.

Results 332 clients evaluated the video-consultations, professionals 535. Clients appreciated the video-consultations on the same positive level as the regular consultations: good contact with professional, enough possibilities to answer all questions and good advice. The professionals also evaluated the videoconsultations positive. The time periods of the video-consultations: $43 \%$ less than 10 minutes, 37\% $10-15$ minutes, $21 \%$ more than 15 minutes.

Conclusion For consultations without the need for physical examinations and no complex requests, video-consultations can provide the same quality as regular consultations and they are appreciated by the clients. Efficiency advantages seem limited if the time needed to organize STI-testing on a distance is included.

Disclosure No significant relationships.

\section{P399 HIV PREVENTION AMONG YOUNG KEY POPULATION: COMMUNITY-BASED STRATEGY OUTCOMES, BRAZIL}

Diego Calixto*, Ana Roberta Pascom, Isabela Pereira, Irene Valderrama, Carina Sousa, Silvia Giugliani, Gilvane Silva, Nara Araújo, Gerson Fernando Pereira. Ministry of Health of Brazil, Department of Surveillance, Prevention and Control of STIS, HIVIAIDS and Viral Hepatitis, Brasilia, Brazil

\subsection{6/sextrans-2019-sti.491}

Background Brazil's HIV epidemic is largely concentrated among key populations whose HIV infection rates can be up to 30 times higher than those observed in the general population. Rapid HIV testing and combination prevention in community settings, delivered by peers, allows for easier access to HIV testing. Our aim is to present the percentage of positive HIV rapid tests and intersectional risk factors of young key populations (YKP) targeted by the 'Live Better Knowing' programme, a nationwide initiative launched by the Brazilian Ministry of Health in close collaboration with NGOs.

Methods Programmatic data were analyzed, collected through Registration form of the Monitoring and Evaluation System Registration Form (SIMAV-Pro), collected from January 1st 2016 from December 31st 2018. 51 Brazilian NGOs administrated the form and offer rapid oral fluid HIV tests (DPP HIV-1/2 Bio-Manguinhos/Fiocruz) and combination prevention packages to sex workers, men who have sex with men (MSM), trans people, people who use drugs and and youngsters in these populations.

Results In total, 88,052 were tested aged from 15 to 24 yo, $69.2 \%$ were nonwhite, $58.9 \%$ reported drug use, $2.6 \%$ commercial sex, and $13.8 \%$ drug use and commercial sex combined. Overall, 49\% reported condom use during their last sexual intercourse and $9 \%$ reported STI symptoms in the last 12 months. The general HIV prevalence found was $1 \%$ and its distribution among women, transvestites, trans women, trans men, MSM, and heterosexual men was $17 \%, 9 \%, 5 \%$, $1 \%, 49 \%$, and $19 \%$ respectively.

Conclusion Community-based rapid HIV testing delivered by peers reached YKP that had not previously accessed HIV testing. Given the combination of drug use and sex work, comprehensive combination prevention services need to be delivered. Our analyses suggest the need to impact behaviors 
related YKP focusing on sex workers, people who use drugs, MSM, and Trans people. These findings support communitybased strategies in public health policies.

Disclosure No significant relationships.

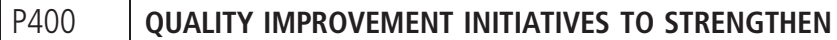 VIRAL SUPPRESSION AMONG ALHIVS IN INSTITUTE OF HUMAN VIROLOGY SITES IN ABUJA}

Henrietta Ezegbe*, Malcolm Steinberg. Simon Fraser University, Health Sciences, Burnaby, Canada

10.1136/sextrans-2019-sti.492

Background Adolescents living with HIV (ALHIVs) in high ALHIV-burden, resource-limited settings like Nigeria have significantly inferior outcomes from antiretroviral therapy. This paper expands on these inferior outcomes including inferior access to and coverage of ART, higher rates of loss to followup, poor adherence, increased needs for psychosocial support and sexual reproductive health services. This study reports on initiatives to identify and bridge gaps in achieving HIV suppression among Nigerian ALHIVs, and ensuring they are not left behind in the UNAIDS 90-90-90 targets.

Methods A retrospective chart review was conducted for ALHIVs aged 10 to 19 years who attended IHVN facilities in Abuja from June 2017-July 2018 with viral loads $\geq 1000$ copies/ml. Quality improvement projects known as 'small tests of change' to improve poor performing areas were initiated. This performance review initiative captured data on a template that highlights the Problem Statement, a Root Cause Analysis, Ideas Intended for Use, an Aims Statement, and a Plan Do Study Act (PDSA). This approach forms the basis of a monitoring cycle to track innovation and outcomes, which may be considered as failed, ongoing, or successfully completed.

Results Inadequate ALHIV suppression was captured as the Problem Statement, the Root Cause Analysis highlighted challenges with - disclosure of HIV status, ART adherence, HIV/ AIDS related stigma and transition to adult services. Some Ideas intended for Use included- Intensified Adherence Counselling, peer mentoring, and youth friendly services, while the Aims Statement was a need to implement initiatives that strengthen ALHIV suppression rates. The Plan Do Study Act (PDSA) stages are currenlty - successfully completed and on going.

Conclusion Attempts to address the needs of ALHIVs are burdened with many challenges, which are magnified in subSaharan Africa where HIV prevalence is high and resources are scarce. These report detail strategies initiated to ensure ALHIVS are not left behind in the UNAIDS 90-90-90 targets. Disclosure No significant relationships.

\section{P401} PROVISION OF ONLINE AND OFFLINE HIV SELF-TESTING KITS FOR STUDENTS ATTENDING UNIVERSITIES: EXPERIENCE FROM SICHUAN, CHINA

${ }^{1}$ Song Fan*, ${ }^{2}$ Run Chen, ${ }^{3}$ Hang Chen, ${ }^{3}$ Caibi Feng, ${ }^{4}$ Xia Jin, ${ }^{5}$ Huachun Zou. ${ }^{1}$ Sun Yat-Sen University, School of Public Health, Guangzhou, China; '2Southwest Medical University, School of Public Health, Luzhou, China; ${ }^{3}$ Luzhou Center for Disease Control and Prevention, Luzhou, China; ${ }^{4}$ AIDS Healthcare Foundation (AHF) China, Beijing, China; ${ }^{5}$ Sun Yat-sen University, School of Public Health (Shenzhen), Guangzhou, China

\subsection{6/sextrans-2019-sti.493}

Background Recent years saw climbing cases of newly HIV infection among students attending universities in China. HIV self-testing (HIVST) has the potential to reach students who do not access facility-based services. We aimed to evaluate the effect of a program providing both online and offline HIVST for students attending universities in China.

Methods HIVST awareness campaigns were conducted in five universities between November 2017 and December 2018 in Luzhou, southwestern China's Sichuan Province. Eligible participants were students attending the five participating universities who were willing to use HIVST. For online HIVST, participants were recruited via social media and provided with a HIVST kit through postal services. For offline HIVST, participants were recruited via campus promotion and provided with a HIVST on site. Participants self-tested their HIV status using a finger-prick HIVST kit with text and pictorial instructions. Testing results were uploaded to online recording system via mobile phones.

Results A total of 649 (122 online, 527 offline) HIVST kits were distributed, of which 490 testing results were returned (82 online and 408 offline). The success rate was high for both online and offline HIVST $(91.5 \%$ vs 93.6\%). All qualified HIVST results were HIV negative. Participants' average age was 19.9 (range 16-29) years. Male students were more likely than their female counterparts to use HIVST (464 vs 26). The reasons for male students to test for HIV were unprotected virginal intercourse with a woman (114, 24.6\%), unprotected anal intercourse with another man (55, 11.9\%). The proportion of MSM in online testers were higher than that in offline testers $(56.4 \%$ vs $43.6 \%)$.

Conclusion This study found high level of qualified results and feedback of HIVST among students attending universities in Luzhou, China. HIVST could be widely implemented to facilitate the HIV testing on campus. Further studies are needed assess the feasibility of its scaling up.

Disclosure No significant relationships.

\section{P402 LARGE DUTCH STUDY REVEALS LOW-EDUCATED YOUNGSTERS ARE AN IMPORTANT TARGET GROUP FOR SEXUAL HEALTH PREVENTION ACTIVITIES}

${ }^{1}$ Kiki Op Den Camp, ${ }^{2}$ Nicole Dukers-Muijrers, ${ }^{1}$ Christian Hoebe* ${ }^{1}$ Geneviève Van Liere. ${ }^{1}$ Public Health Service South Limburg, Maastricht University Medical Center (MUMC), Sexual Health, Infectious Diseases and Environmental Health, Medical Microbiology, Care and Public Health Research Institute (CAPHRI), Heerlen, Netherlands; ${ }^{2}$ Public Health Senvice South Limburg, Sexual Health, Infectious Diseases and Environmental Health, Heerlen, Netherlands

\subsection{6/sextrans-2019-sti.494}

Background Youngsters under the age of twenty-five have an increased risk for sexual health problems. Therefore, a large population based online survey on the sexual health of 